\title{
MicroRNA-127-3p acts as a tumor suppressor in epithelial ovarian cancer by regulating the BAG5 gene
}

\author{
LINLIN BI $^{1}$, QIAN YANG ${ }^{1}$, JIANI YUAN $^{1}$, QING MIAO $^{3}$, LINRUI DUAN $^{1}$, \\ FENG $\mathrm{LI}^{2}$ and SIWANG WANG ${ }^{1}$ \\ ${ }^{1}$ Department of Natural Medicine, School of Pharmacy, The Fourth Military Medical University, Xi'an, Shaanxi 710032; \\ ${ }^{2}$ Department of Traditional Chinese Medicine, Xijing Hospital of The Fourth Military Medical University, Xi'an, \\ Shaanxi 710032; ${ }^{3}$ Department of Pharmacy, PLA 401 Hospital, Qingdao, Shandong 266071, P.R. China
}

Received February 3, 2016; Accepted March 15, 2016

DOI: $10.3892 /$ or.2016.5055

\begin{abstract}
In the present study, the tumor-suppressive role of microRNA-127-3p (miR-127-3p) in epithelial ovarian cancer (EOC) was elucidated. Expression of miR-127-3p was examined by quantitative RT-PCR (qRT-PCR) in 9 EOC cell lines and clinical samples from 13 EOC patients. EOC cell lines, OVCAR-3 and Caov-3, were transduced with a lentivirus to overexpress endogenous miR-127-3p. The tumor-suppressive effects of miR-127-3p on EOC proliferation, bufalin sensitivity, invasion and in vivo growth were investigated through proliferation, bufalin sensitivity wound-closure and in vivo tumorigenicity assays, respectively. In addition, luciferase reporter assay and $\mathrm{qRT}$-PCR were conducted to verify whether the Bcl-2-associated athanogene 5 (BAG5) gene was the downstream target of miR-127-3p in EOC. BAG5 was subsequently upregulated in the OVCAR-3 and Caov-3 cells to examine its functional correlation with miR-127-3p regulation in EOC. The results revealed that in both EOC cell lines and EOC tumor tissues, miR-127-3p was downregulated. Lentiviral-mediated miR-127-3p overexpression exerted tumor-suppressive effects in OVCAR-3 and Caov-3 cells by reducing in vitro proliferation and invasion, increasing bufalin sensitivity, and inhibiting in vivo tumor growth. miR-127-3p directly regulated the BAG5 gene in EOC. Subsequent BAG5 upregulation ameliorated the tumor-suppressive effects of miR-127-3p overexpression in EOC. In conclusion, miR-127-3p
\end{abstract}

Correspondence to: Dr Feng Li, Department of Traditional Chinese Medicine, Xijing Hospital of Fourth Military Medical University, 169 Changle West Road, Xi'an, Shaanxi 710032, P.R. China

E-mail: feng_li3@aol.com

Dr Siwang Wang, Department of Natural Medicine, School of Pharmacy, Fourth Military Medical University, 169 Changle West Road, Xi'an, Shaanxi 710032, P.R. China

E-mail: wangsiwang201@163.com

Key words: epithelial ovarian cancer, miR-127-3p, BAG5, cancer proliferation, cancer invasion functions as a tumor suppressor in EOC, and its influence on EOC is directly through regulation of BAG5.

\section{Introduction}

Epithelial ovarian cancer (EOC) is one of the most malignant gynecological cancers worldwide $(1,2)$. In the United States alone, there were more than 20,000 estimated new cases, and more than 14,000 deaths due to EOC (3). Both genetic and epigenetic factors may contribute significantly to the origin of EOC (2). Despite numerous efforts, the exact mechanisms of EOC pathogenesis are fundamentally unknown, and methods for early diagnosis and novel therapies are largely lacking. Thus, it is critical to explore the molecular mechanisms underlying EOC carcinogenesis, development and metastasis to seek novel therapeutic targets for patients with EOC.

MicroRNAs (miRNAs) are a group of small-length (18-22 nucleotides long) non-coding RNAs that bind the complementary sites of the 3'-untranslated region (3'-UTR) of target genes to induce gene inhibition and protein degradation $(4,5)$. miRNAs have been shown to play critical roles in human cancer, acting as either oncogenes or tumor suppressors $(6,7)$. In human EOC, both upregulated and downregulated miRNAs have been discovered (8), suggesting that the regulation of miRNAs in EOC may be complex, as well as their associated signaling pathways. MicroRNA-127-3p (miR-127-3p), also referred to as miR-127, was often found to be downregulated in human tumors and acts as a tumor suppressor in breast and gastric cancer $(9,10)$. miR-127-3p was also found to be downregulated in clinical samples from EOC patients $(11,12)$. Yet, the functional mechanisms of miR-127-3p have never been elucidated in EOC.

In the present study, we evaluated the expression pattern of miR-127-3p in both EOC cell lines and EOC tumor samples. Then, we hypothesized that miR-127-3p may act as a tumor suppressor in EOC as in other human cancers, and tested this hypothesis by endogenously overexpressing miR-127-3p in EOC cell lines, OVCAR-3 and Caov-3, through lentiviral transduction. The functional effects of miR-127-3p overexpression on EOC proliferation, drug (bufalin) sensitivity, invasion and in vivo tumorigenicity were then carefully evaluated. Furthermore, we hypothesized that the 
Bcl-2-associated athanogene 5 (BAG5) gene is the downstream target of miR-127-3p in EOC. We then tested this hypothesis through dual-luciferase reporter assay and qRT-PCR. Subsequently, BAG5 was upregulated in OVCAR-3 and Caov-3 cells, and its interaction with miR-127-3p overexpression on regulating EOC was further investigated.

\section{Materials and methods}

Statement of ethics. All experimental protocols were approved by the ethics committees at the participating hospitals. All procedures were conducted in accordance to the principles of the Declaration of Helsinki, and Local and National Medical Practice Laws. Consent forms were signed by all participating patients.

Cell lines and patients. In this study, five EOC cell lines, SKOV-3, OVCAR-3, Caov-3, ES-2, PA-1 and a non-tumorigenic human-derived ovarian cell line, HS-832, were obtained from the American Type Culture Collection (ATCC; Manassas, VA, USA). Four EOC cell lines, MCAS, OVCA432, OVCA429 and PEO4 were obtained from the Cell Bank of the Type Culture Collection of the Chinese Academy of Sciences (Shanghai, China). All cells were maintained in RPMI-1640 medium supplemented with $10 \%$ fetal bovine serum (FBS) (both from Thermo Fisher Scientific, USA) in a tissue culture chamber supplied with $95 \% \mathrm{CO}_{2}$ and $5 \% \mathrm{CO}_{2}$ at $37^{\circ} \mathrm{C}$. The clinical samples of paired EOC tumors (tumor) and their adjacent normal ovarian epithelial tissues (normal) were surgically obtained from 13 patients between June 2012 to December 2015. Clinical samples were snap-frozen in liquid nitrogen and stored in an $-80^{\circ} \mathrm{C}$ bio-freezer (Forma Scientific, USA) until processed.

RNA isolation and quantitative RT-PCR. Total RNA was isolated from the EOC cell lines or EOC patient clinical samples using an RNeasy Mini kit (Qiagen, USA) according to the manufacturer's protocol. Using 100 ng RNA from each sample, cDNA was reversely synthesized with a TaqMan Reverse Transcription kit (Applied Biosystems, USA). Quantitative RT-PCR (qRT-PCR) was conducted on an ABI Prism 7900 Sequence detection system (Applied Biosystems). For BAG5 gene detection, a SYBR Green PCR Master Mix kit (Applied Biosystems) was conducted with the following reaction conditions, $95^{\circ} \mathrm{C}$ for $15 \mathrm{~min} ; 38$ cycles of $95^{\circ} \mathrm{C}$ for $30 \mathrm{sec}, 58^{\circ} \mathrm{C}$ for $40 \mathrm{sec}$, and $70^{\circ} \mathrm{C}$ for $30 \mathrm{sec}$. $18 \mathrm{~S}$ was used as a loading control. For miR-127-3p detection, a TaqMan miRNA assay (Applied Biosystems) was conducted with the following reaction conditions, $95^{\circ} \mathrm{C}$ for $15 \mathrm{~min}$; and 38 cycles of $95^{\circ} \mathrm{C}$ for $30 \mathrm{sec}$ and $62^{\circ} \mathrm{C}$ for $40 \mathrm{sec}$. U6 snRNA was used as a loading control. All reactions were conducted in biological triplicates. Relative gene expression levels were measured as fold changes using the $2^{-\Delta \Delta C t}$ method.

miR-127-3p overexpression. A lentiviral vector expressing miR-127-3p mimics (L-miR127-Mimic), and a control lentivirus (L-C) were obtained from RiboBio Biotech (China). To overexpress hsa-miR-127-3p in EOC cell lines, $5 \times 10^{5}$ OVCAR-3 or Caov-3 cells were transduced with L-miR127-Mimic, along with a multiplicity of infection of 10 and $5 \mu \mathrm{g} / \mathrm{ml}$ Polybrene for
$4 \mathrm{~h}$. The control EOC cells were transduced with L-C. After 3 washes ( 5 min each time), the EOC cells were replenished with fresh culture medium and maintained for $48 \mathrm{~h}$. After that, floating cells were aspirated. Healthy cells were suspended, re-seeded and readied for the next experiments.

Cancer proliferation assay. Five hundred OVCAR-3 or Caov-3 cells were seeded in a 96-well plate. The growth of EOC cells was measured by a CellTiter $96^{\circledR}$ Aqueous One cell proliferation assay (Promega, Madison, WI, USA) according the manufacturer's protocol. Briefly, proliferation solution (20 $\mu \mathrm{l})$ was added into the cell culture for $1 \mathrm{~h}$ at $0,1,2,3$ and 4 days. The absorbance was measured at $490 \mathrm{~nm}$.

Bufalin sensitivity assay. OVCAR-3 or Caov-3 cells were incubated with bufalin at various concentrations $(0,0.1,0.5$, 1,10 and $100 \mathrm{ng} / \mathrm{ml}$ ) for $48 \mathrm{~h}$. The sensitivity of the EOC cells to bufalin was estimated by relative cell viability, which was measured by proliferation assay and then normalizing the absorbance values to the ones under control conditions (no bufalin treatment).

Invasion assay. In vitro invasive capability was measured by a wound closure assay. Briefly, 5x10 $0^{3}$ OVCAR-3 or Caov-3 cells were seeded in 96-well plates. Once confluent, the cells were incubated with $10 \mu \mathrm{g} / \mathrm{ml}$ mitomycin (Sigma-Aldrich, USA) for $2 \mathrm{~h}$ to stop proliferation. Then, a 96-pin wound-maker (Essen Biosciences, UK) was used to create defined wound areas. Phase-contrast images were captured immediately $(0 \mathrm{~h})$ and $24 \mathrm{~h}$ after wound creation. The invasive capability was determined by measuring the reduction on the wound area between 0 and $24 \mathrm{~h}$ using ImageJ software (NIH, Bethesda, MA, USA).

Tumorigenicity assay. OVCAR-3 cells transduced with L-miR127-Mimic or L-C were subcutaneously injected into both flanks of four adult athymic nude mice $\left(1 \times 10^{6} /\right.$ injection in 8-week old mice). Tumor volume $\left(\mathrm{mm}^{3}\right)$ was monitored weekly for 5 weeks, using the formula $\mathrm{Lx} \mathrm{W}^{2} / 2$, in which $\mathrm{L}$ and $\mathrm{W}$ are the longest and shortest diameters respectively of the tumor. After 5 weeks, the mice were sacrificed. Tumors from both flanks were extracted from 4 mice (M1-M4) for visual examination.

Luciferase reporter assay. Wild-type and mutant 3'-UTRs of the human BAG5 gene were cloned into the psiCHECK2 luciferase vector (Promega). HEK293T cells were seeded in 6-well plates and co-transfected with BAG5-WildType, BAG5-Mutant or Renilla luciferase vectors, and L-C or L-miRMimic lentiviruses. Forty-eight hours after co-transfection, a dual-luciferase reporter assay (Promega) was conducted according to the manufacturer's protocol. Relative luciferase activities (against Renilla activity) were normalized to the activity with L-C transfection.

BAG5 upregulation assay. The whole sequence of the human BAG5 gene was cloned into a eukaryotic expression vector pcDNA3.1 (Life Technologies, USA) to generate a BAG5 overexpressing vector (pcDNA/BAG5). An empty pcDNA3.1 vector (pcDNA/+) was used as the control. OVCAR-3 and Caov-3 cells were then transfected with pcDNA/BAG5 or 
A

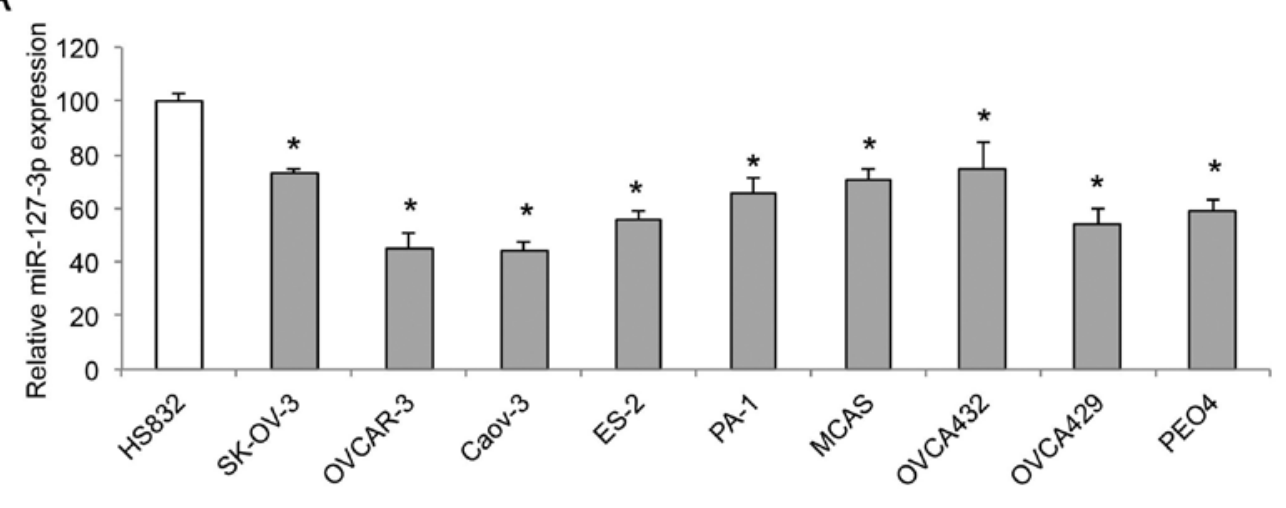

B
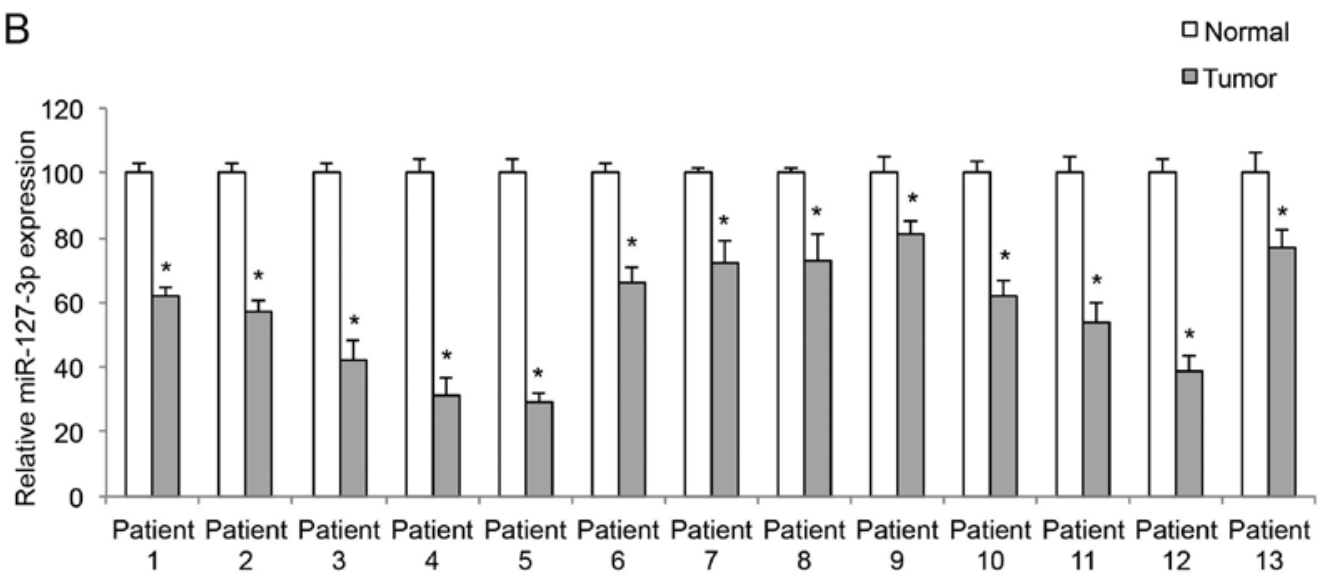

Figure 1. miR-127-3p is downregulated in EOC. (A) Quantitative RT-PCR (qRT-PCR) was conducted to compare the gene levels of miR-127-3p among 9 EOC cell lines, SK-OV-3, OVCAR-3, Caov-3, ES-2, PA-1, MCAS, OVCA432, OVCA429, PEO4, and a non-tumorigenic human-derived ovarian cell line, HS-832 ("P<0.05). (B) qRT-PCR was conducted to compare paired clinical samples, EOC tumors (tumor) vs. adjacent normal ovarian tissues (normal), from 13 EOC patients $\left({ }^{*} \mathrm{P}<0.05\right)$.

pcDNA/+ using Lipofectamine 2000 reagent (Thermo Fisher Scientific). Forty-eight hours after transfection, qRT-PCR was conducted to verify upregulation efficiency.

Statistical analysis. All assays were repeated at least three times. Results are presented as means \pm standard errors. Statistic analyses were conducted using the Student's t-test on Windows-based SPSS software (SPSS 11.0; SPSS, Inc., USA). Statistical differences were significant at $\mathrm{P}<0.05$.

\section{Results}

miR-127-3p is downregulated in EOC cell lines and EOC tumors. The expression of miR-127-3p was compared among nine EOC cell lines, SK-OV-3, OVCAR-3, Caov-3, ES-2, PA-1, MCAS, OVCA432, OVCA429, PEO4 and a non-tumorigenic human-derived ovarian cell line, HS-832 by qRT-PCR. It showed that miR-127-3p was markedly downregulated in all examined EOC cell lines, when compared with its level in the HS-832 cells (Fig. 1A, P<0.05). The expression of miR-127-3p was also compared, by qRT-PCR, between paired EOC tumors (T) and adjacent normal ovarian epithelial tissues (normal) in 13 patients with EOC. The result demonstrated that miR-127-3p was downregulated in the EOC tumors compared to the level in the normal ovarian tissues (Fig. $1 \mathrm{~B}, \mathrm{P}<0.05$ ).
miR-127-3p overexpression inhibits cancer growth and increases bufalin sensitivity in EOC. Two of the EOC cell lines, OVCAR-3 and Caov-3, were transduced with L-miR127-Mimic to overexpress endogenous miR-127-3p. The control EOC cells were transduced with an empty control lentivirus, L-C. After transduction, qRT-PCR was conducted to compare miR-127-3p expression between the EOC cells transduced with L-miR127-mimic or L-C. Transduction of L-miR127-Mimic, as compared to L-C, markedly upregulated endogenous miR-127-3p in both the OVCAR-3 and Caov-3 cells (Fig. 2A, P<0.05).

We then compared cancer cell growth in the lentiviraltransduced EOC cells. The proliferation assay showed that miR-127-3p overexpression significantly inhibited cancer growth in the OVCAR-3 cells 2 days after the onset of the proliferation assay, and in Caov-3 cells $24 \mathrm{~h}$ after the onset of the proliferation assay (Fig. $2 \mathrm{~B}, \mathrm{P}<0.05$ ). We also assessed the effect of miR-127-3p on EOC bufalin sensitivity. miR-127-3p overexpression significantly increased bufalin sensitivity by reducing cell viability in both the OVCAR-3 and Caov-3 cells (Fig. 2C, $\mathrm{P}<0.05$ ).

miR-127-3p overexpression reduces cancer cell invasive capability in EOC. In the lentiviral-transduced OVCAR-3 and Caov-3 cells, a wound-closure assay was conducted 
A

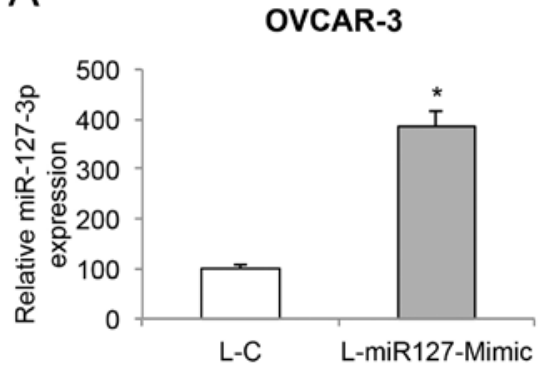

B
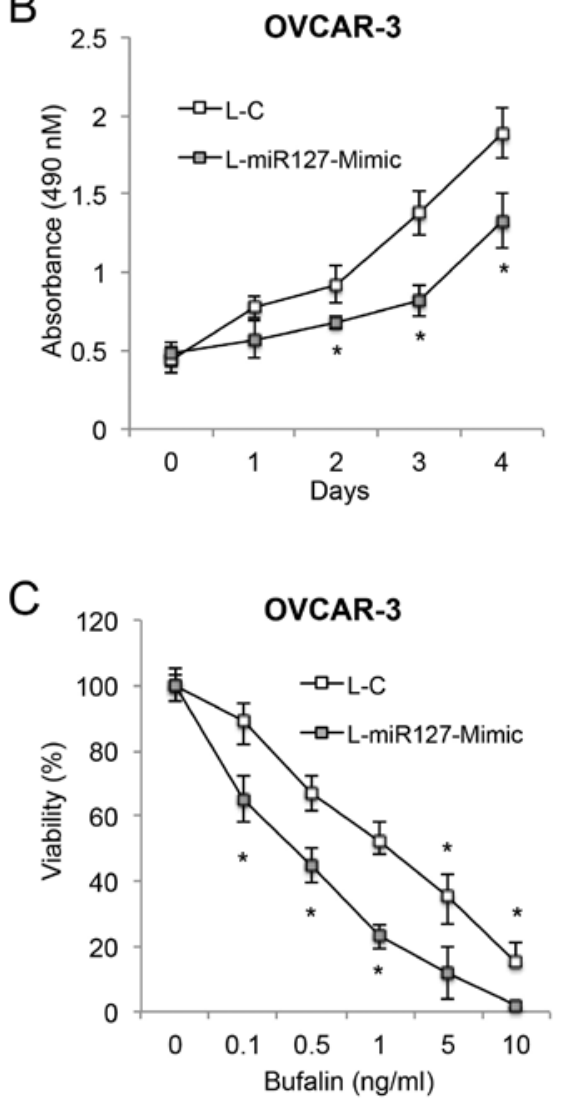

Caov-3
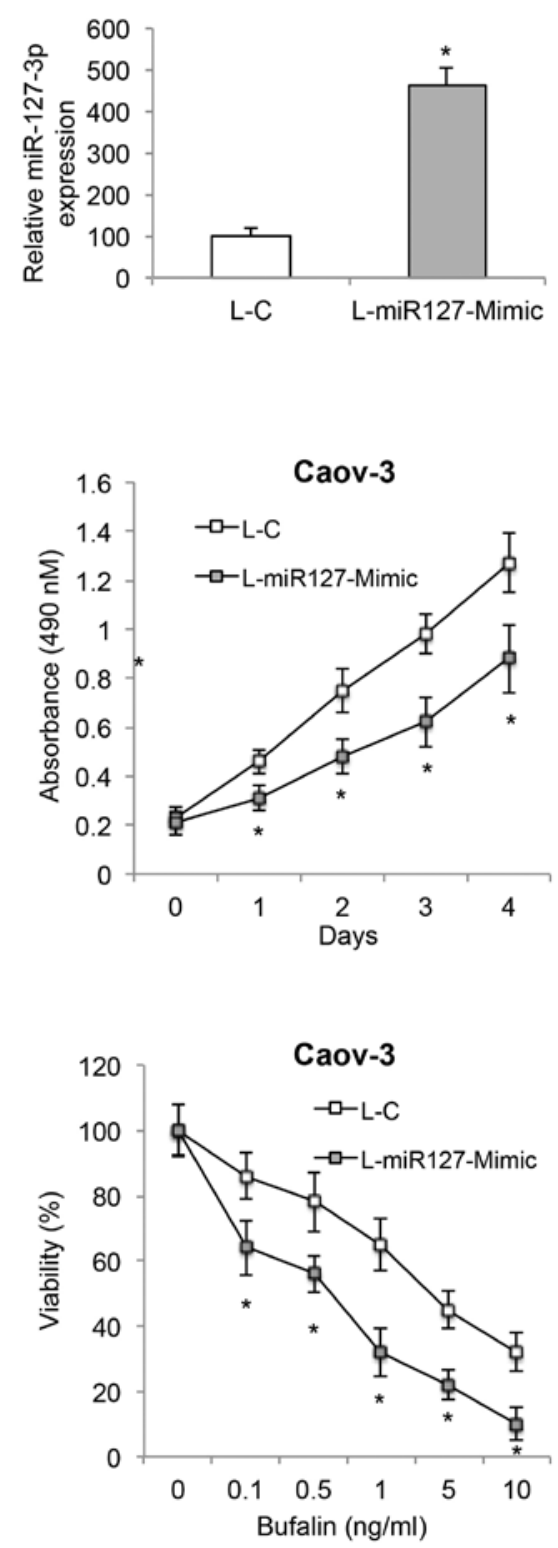

Figure 2. miR-127-3p overexpression reduces proliferation and increases bufalin sensitivity in EOC. (A) OVCAR-3 and Caov-3 cells were transduced with a miR-127-3p-mimics lentivirus (L-miR127-Mimic), or a control lentivirus (L-C). qRT-PCR was conducted to compare endogenous miR-127-3p expression between EOC cells transduced with L-miR127-Mimic and EOC cells transduced with L-C ( $\mathrm{P}<0.05$ ). (B) After lentiviral transduction, a proliferation assay was conducted to compare cancer cell growth between EOC cells transduced with L-miR127-Mimic and EOC cells transduced with L-C ( $\mathrm{P}<0.05)$. (C) After lentiviral transduction, OVCAR-3 and Caov-3 cells were treated with bufalin $(0,0.1,0.5,1,5$ and $10 \mathrm{ng} / \mathrm{ml})$ for $48 \mathrm{~h}$. Cell viability was compared between cells transduced with L-miR127-Mimic and cells transduced with L-C $\left({ }^{*} \mathrm{P}<0.05\right)$.

to assess the effect of miR-127-3p overexpression on EOC invasion. Images are shown for the EOC cells immediately after wound creation $(0 \mathrm{~h})$, and $24 \mathrm{~h}$ later. A reduced degree of wound closure was observed in the OVCAR-3 and Caov-3 cells transduced with L-miR127-Mimic, than that in the cells transduced with L-C (Fig. 3, left). Subsequent quantification confirmed that miR-127-3p overexpression markedly reduced the invasive capabilities in both the OVCAR-3 and Caov-3 cells (Fig. 3, right, $\mathrm{P}<0.05$ ).

miR-127-3p overexpression inhibits in vivo tumorigenicity of EOC. We then investigated whether miR-127-3p overexpression had an effect on the in vivo growth of EOC.
L-miR127-Mimic-transduced OVCAR-3 cells $\left(1 \times 10^{6}\right)$ were subcutaneously injected into the left flanks of 8 -week-old athymic nude mice $(n=4)$. L-C-transduced OVCAR-3 cells $\left(1 \times 10^{6}\right)$ were subcutaneously injected into the right flanks of the nude mice. The in vivo growth curves were monitored weekly, for 5 consecutive weeks, by measuring tumor volume $\left(\mathrm{mm}^{3}\right)$. The result showed that miR-127-3p overexpression markedly inhibited in vivo EOC tumor growth (Fig. 4A, $\mathrm{P}<0.05$ ). At the end of the tumorigenicity assay, tumors were extracted from both flanks of the mice, and compared. L-miR-127-Mimic-transduced EOC tumors were significantly smaller than the L-C-transduced tumors in all 4 mice (Fig. 4B). 
A
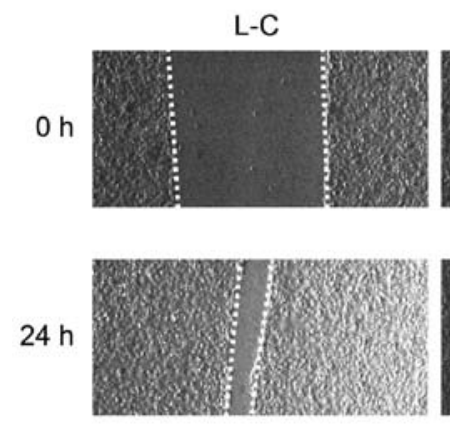

B
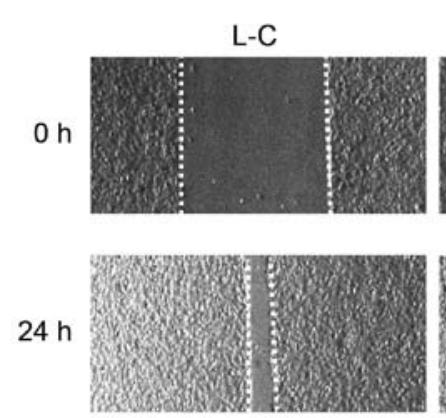
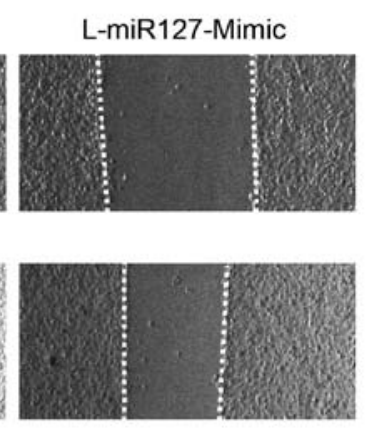

Caov-3
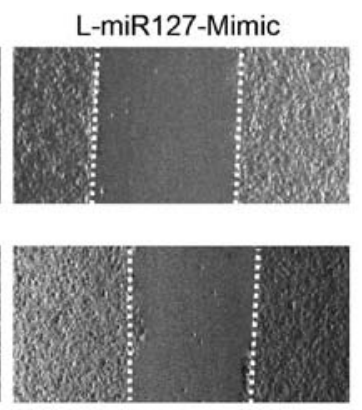
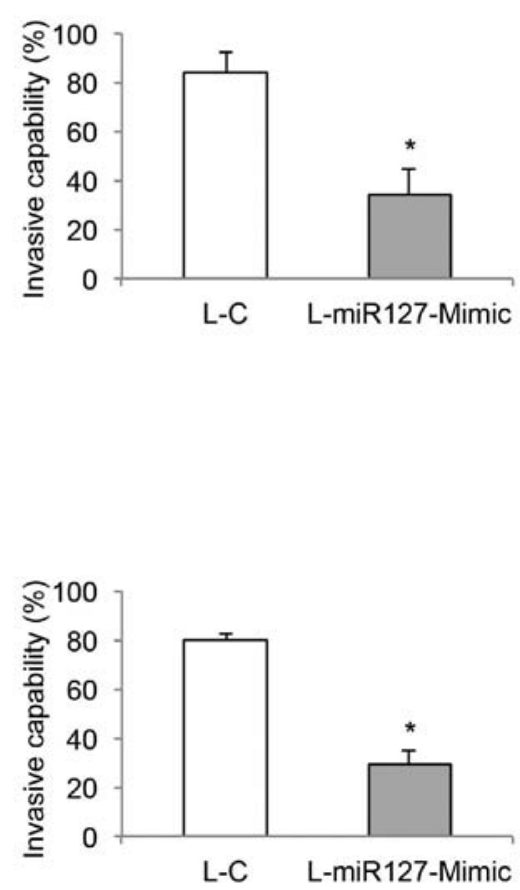

Figure 3. miR-127-3p overexpression reduces cancer invasion in EOC. After lentiviral transduction, OVCAR-3 (A) and Caov-3 cells (B) were seeded in 96-well plates and a wound-closure assay was performed. Phase-contrast images were captured at 0 and $24 \mathrm{~h}$ after wound creation (left). The invasive capability was determined by measuring the reduction in wound area between 0 and $24 \mathrm{~h}$ in EOC cells (right, ${ }^{*} \mathrm{P}<0.05$ ).

A

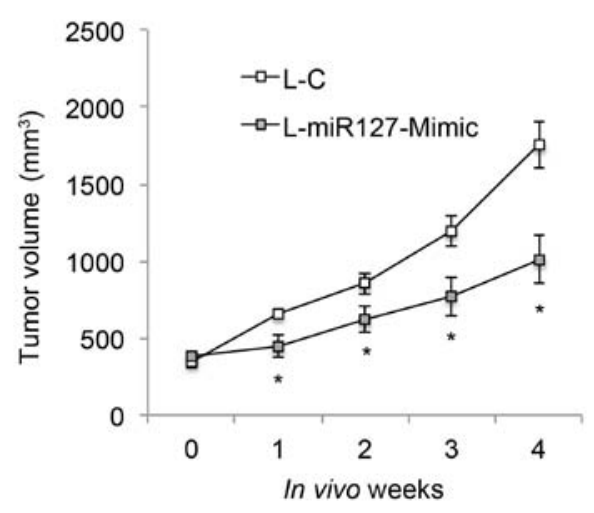

B

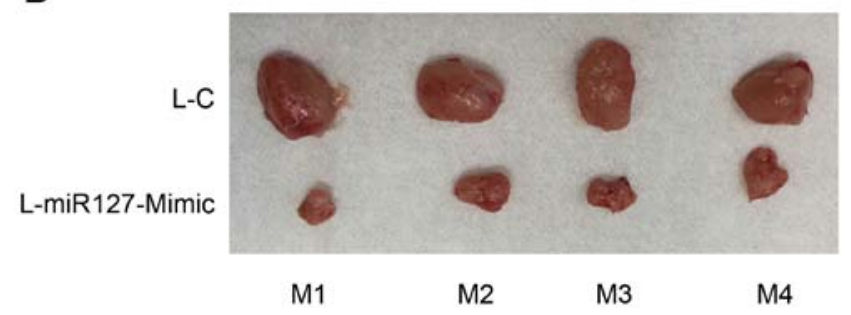

Figure 4. miR-127-3p overexpression reduces EOC growth in vivo. (A) Lentiviral-transduced OVCAR-3 cells were subcutaneously injected into both flanks of four athymic null mice ( $1 \times 10^{6}$ cells per flank per mouse). For five consecutive weeks, tumor volumes $\left(\mathrm{mm}^{3}\right)$ were measured weekly using the formula, $\mathrm{L} \mathrm{x} \mathrm{W} / 2$, in which $\mathrm{L}$ and $\mathrm{W}$ stand for the longest and shortest diameters of the tumor. Tumor volume was compared between tumors transduced with L-miR127-Mimic and tumors transduced with L-C ( $\left.{ }^{*} \mathrm{P}<0.05\right)$. (B) At the end of the tumorigenicity assay, tumors were extracted from 4 mice (M1-M4) and compared.
BAG5 is directly associated with miR-127-3p overexpression in EOC. Since we demonstrated that miR-127-3p overexpression has a tumor-suppressive effect on EOC, we then explored the molecular target of miR-127-3p. Through web-search on miRNA targets, including miRDB (www. mirdb.org) and TargetScan Human (www.targetscan.org), we found that the BAG5 gene is a candidate downstream target gene of miR-127-3p (Fig. 5A). We then used a dual-luciferase reporter assay to verify this hypothesis. HEK293T cells were co-transfected with BAG5-Wild-type, BAG5-Mutant or Renilla luciferase vectors, and L-C or L-miR127-Mimic lentiviruses. Forty-eight hours after co-transfection, quantification demonstrated that the relative luciferase activities between L-C and L-miR127-Mimic transfections significantly differed in cells transfected with BAG5-WildType luciferase reporter, but in cells transfected with BAG5-Mutant luciferase reporter (Fig. 5B, $\mathrm{P}<0.05 ; \mathrm{P}>0.05$ ), thus confirming that BAG5 is the target gene of miR-127-3p. In addition, we examined the mRNA levels of BAG5 in miR-127-3p overexpressed OVCAR-3 and Caov- 3 cells. The result of qRT-PCR demonstrated that miR-127-3p overexpression significantly downregulated endogenous BAG5 in the EOC cells (Fig. 5C, $\mathrm{P}<0.05$ ).

BAG5 upregulation induces opposite effects opposite to those of miR-127-3p overexpression in EOC. In order to elucidate the function of the BAG5 gene in miR-127-3p-mediated EOC regulation, we transfected L-miR127-Mimic-transduced OVCAR-3 and Caov-3 cells with a BAG5-overexpressing vector pcDNA/BAG5, or a control vector pcDNA/+. Forty- 
A

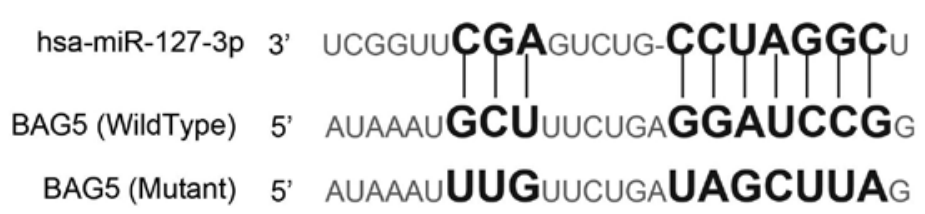

B
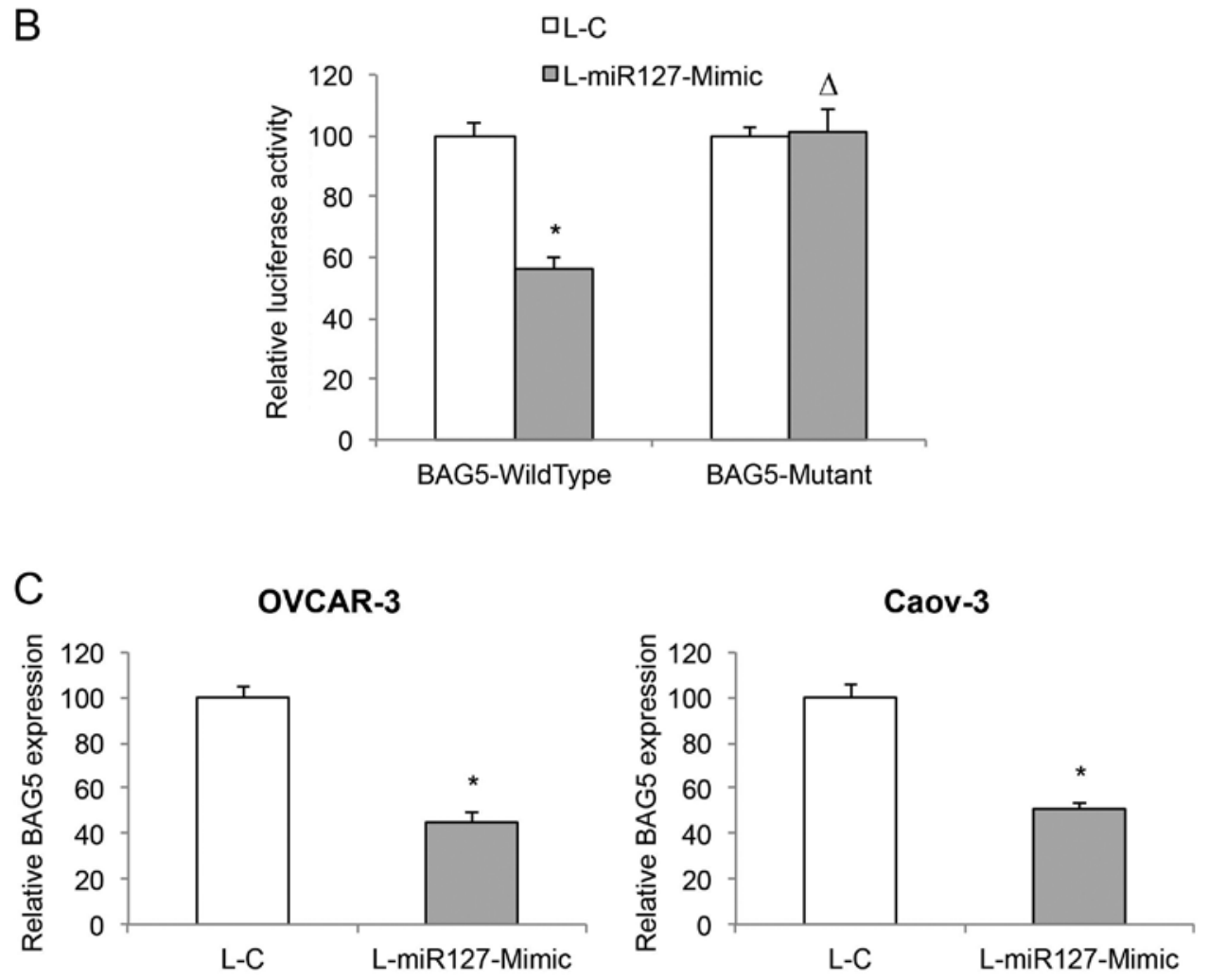

Figure 5. BAG5 is targeted by miR-127-3p in EOC. (A) The complimentary association of hsa-miR-127-3p and 3'-UTR of the human BAG5 gene (wildtype) is shown. A mutant BAG5 3'-UTR, with mutated hsa-miR-127-3p binding sequence, is also shown. (B) HEK293T cells were co-transfected with BAG5-Wild-type, BAG5-Mutant or Renilla luciferase vectors, and L-C or L-miR127-Mimic lentiviruses for $48 \mathrm{~h}$. Relative luciferase activities were measured by a dual-luciferase reporter assay and normalized to the values with $\mathrm{L}-\mathrm{C}$ transfection $\left({ }^{*} \mathrm{P}<0.05,{ }^{\Delta} \mathrm{P}>0.05\right)$. (C) Expression of the BAG5 gene was compared by qRT-PCR in the OVCAR-3 and Caov-3 cells between those transduced with L-miR127-Mimic and those transduced with L-C ("P<0.05).

eight hours after transfection, qRT-PCR demonstrated that endogenous BAG5 mRNAs were significantly upregulated in the EOC cells transfected with pcDNA/BAG5 than the expression level in the EOC cells transfected with pcDNA/+ (Fig. 6A, P<0.05).

We then examined the effect of BAG5 upregulation on cancer growth, bufalin sensitivity and invasive capabilities in those double-transfected EOC cells. The proliferation assay showed that BAG5 upregulation revitalized cancer growth in the miR-127-3p-overexpressing EOC cells (Fig. 6B, $\mathrm{P}<0.05$ ). The bufalin sensitivity assay demonstrated that BAG5 upregulation ameliorated bufalin sensitivity in the miR127-3p-overexpressing EOC cells (Fig. 6C, P<0.05). Furthermore, wound-closure assay showed that BAG5 upregulation also significantly restored invasive capability in the miR-127-3p-overexpressing EOC cells (Fig. 7, $\mathrm{P}<0.05$ ). Thus, our experiments using the double-transfected EOC cells clearly demonstrated that BAG5 upregulation induced effects opposite those of miR-127-3p overexpression on EOC growth, bufalin sensitivity and invasion.

\section{Discussion}

MicroRNAs have been shown to play important roles in EOC regulation, acting as either oncogenes or tumor suppressors $(8,12)$. MicroRNA-127-3p has been identified as a tumor suppressor in other human cancers $(9,10,13)$, and has been shown to be downregulated in EOC tumors $(11,12)$. Yet, the exact functional roles of miR-127-3p in EOC are elusive. In our study, we first used quantitative method, qRT-PCR, to assess the expression pattern of miR-127-3p. We verified that miR-127-3p was downregulated in EOC cell lines in vitro, as well as in in vivo clinical samples of EOC tumors.

Since all evidence points to a downregulated expression pattern of miR-127-3p in EOC, we took further step to explore the functional mechanism of miR-127-3p. We thus created two EOC cell lines, OVCAR-3 and Caov-3, with stable miR127-3p overexpression. Subsequent functional experiments demonstrated that miR-127-3p acted as a tumor suppressor in $\mathrm{EOC}$, as overexpression of miR-127-3p inhibited EOC proliferation, drug (bufalin) sensitivity, invasion and in vivo tumor 
A

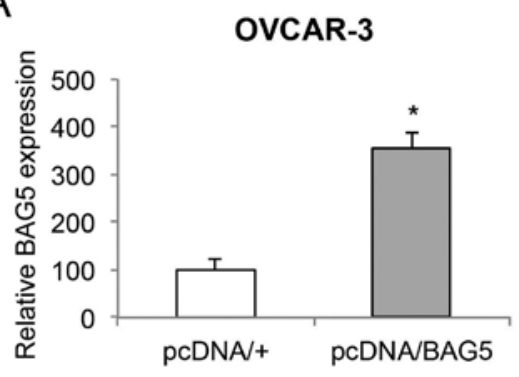

B

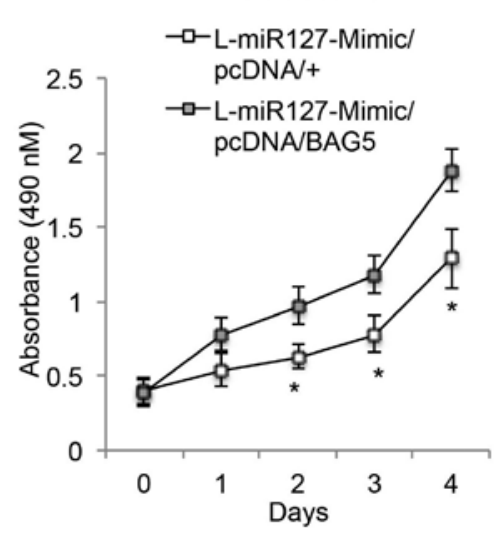

C

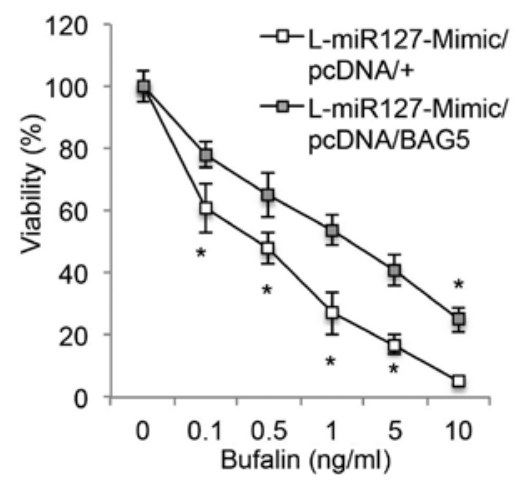

Caov-3

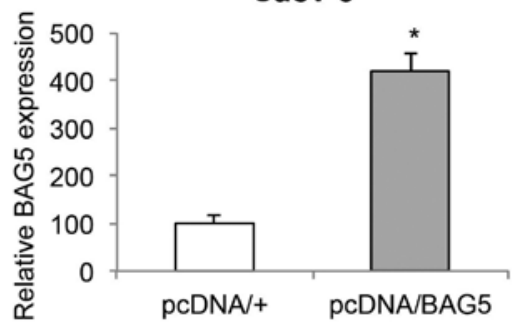

Caov-3

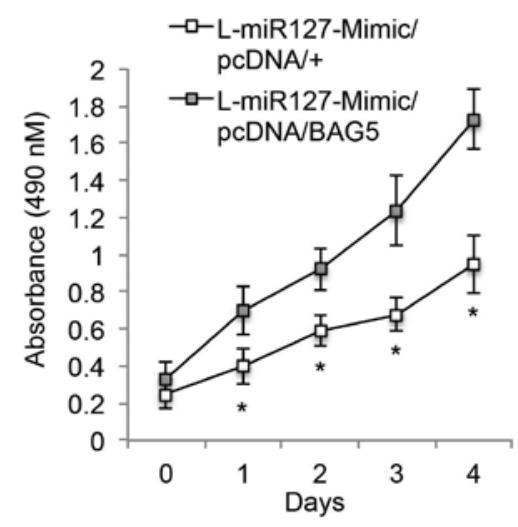

Caov-3

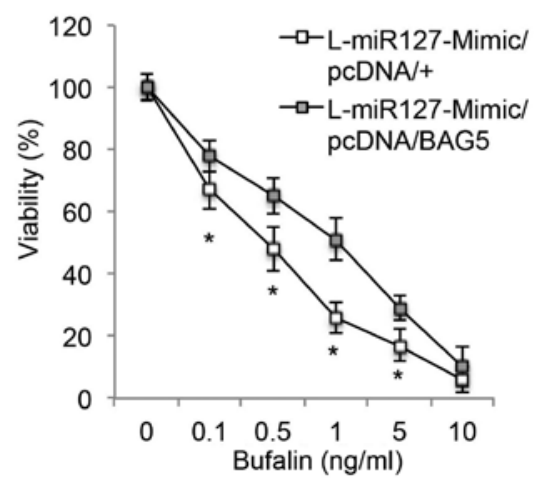

Figure 6. BAG5 upregulation reverses the effects of miR-127-3p overexpression on proliferation and bufalin sensitivity in EOC. (A) In the L-miR127Mimic-transduced OVCAR-3 and Caov-3 cells, second transfection of the BAG5-overexpressing vector (pcDNA/BAG5) or an empty vector (pcDNA/+) was performed for $48 \mathrm{~h}$. qRT-PCR was conducted to compare endogenous BAG5 mRNA expression ( $\left.{ }^{*} \mathrm{P}<0.05\right)$. (B) After second-transfection of the miR-127-3poverexpressing EOC cells, a proliferation assay was conducted to compare cancer cell growth between the cells transfected with pcDNA/BAG5 and cells transfected with pcDNA/+ $\left({ }^{*} \mathrm{P}<0.05\right)$. (C) A bufalin sensitivity assay was conducted to compare the cell viability between miR-127-3p-overexpressing EOC cells which were transfected with pcDNA/BAG5 and those which were transfected with pcDNA/+ $\left({ }^{*} \mathrm{P}<0.05\right)$.

development. Therefore, collectively $(9,10,13)$, it may well demonstrate that miR-127-3p predominantly acts as a tumor suppressor across various cancer types.

Through investigations on breast and bladder cancer, it was found that the major molecular target of tumorsuppressive miR-127-3p was a zinc-finger repressor gene BCL6 $(9,14)$. However, the results of our study revealed that the BAG5 gene, a novel candidate, was likely the target gene of miR-127-3p in ovarian cancer. Our dual-luciferase reporter assay demonstrated that has-miR-127-3p did bind to the complimentary sites on the human BAG5 gene. Our qRT-PCR analysis also showed that the BAG5 gene was directly downregulated by $\mathrm{miR}-127-3 \mathrm{p}$ overexpression in the EOC cell lines, OVCAR-3 and Caov-3. Moreover, our functional experiments provided unambiguous evidence showing that upregulation of the BAG5 gene inversely regulated the tumor-suppressive effects of miR-127-3p overexpression in EOC. Notably, BAG5 belongs to the gene family of BCL2, which was reported to be involved in chromosomal translocations of the BCL6 gene $(15,16)$. Therefore, future investigations focusing on trans-locational evidence of BCL2/BCL6 in patients with EOC may help to identify whether BAG5 and BCL6 are interactively involved in the microRNA regulation in EOC. 
A OVCAR-3
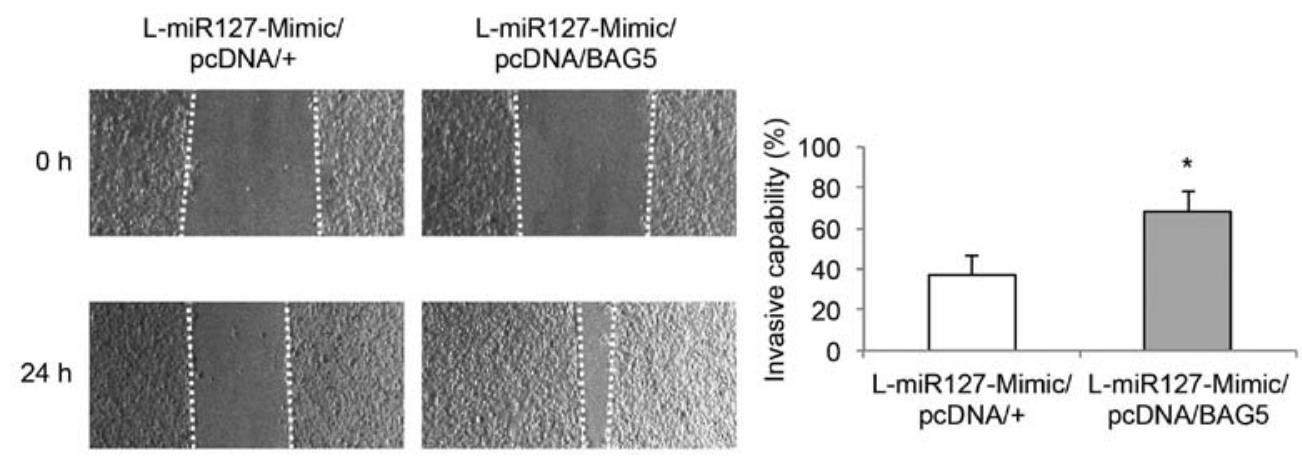

B
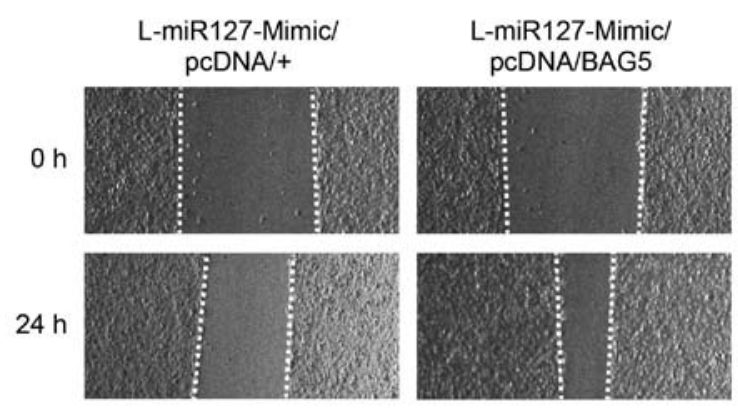

Caov-3

Figure 7. BAG5 upregulation reverses the effects of miR-127-3p overexpression on cancer invasion in EOC. miR-127-3p-overexpressing OVCAR-3 and Caov-3 cells were transfected with either pcDNA/BAG5 or pcDNA/+. OVCAR-3 (A) and Caov-3 cells (B) were re-plated in 96-well plates and their invasive capabilities were examined by a wound-closure assay. Phase-contrast images were captured at 0 and $24 \mathrm{~h}$ after wound creation (left). The invasive capability was determined by measuring the reduction in wound area between 0 and $24 \mathrm{~h}$ in the EOC cells (right, ${ }^{*} \mathrm{P}<0.05$ ).

In conclusion, we discovered novel regulatory mechanism of miR-27-3p acting as a tumor suppressor in EOC. Moreover, we discovered a novel molecular target, BAG5 gene, to be the downstream target of miR-127-3p in regulating EOC development. Our results further the knowledge of epigenetic regulations in EOC, as well as help to development new targets for early diagnosis and optimal therapies for patients with EOC.

\section{References}

1. Jazaeri AA: Molecular profiles of hereditary epithelial ovarian cancers and their implications for the biology of this disease. Mol Oncol 3: 151-156, 2009.

2. Al Bakir M and Gabra H: The molecular genetics of hereditary and sporadic ovarian cancer: Implications for the future. Br Med Bull 112: 57-69, 2014

3. Siegel RL, Miller KD and Jemal A: Cancer statistics, 2015. CA Cancer J Clin 65: 5-29, 2015.

4. Alvarez-Garcia I and Miska EA: MicroRNA functions in animal development and human disease. Development 132: 4653-4662, 2005.

5. Zhang B, Wang Q and Pan X: MicroRNAs and their regulatory roles in animals and plants. J Cell Physiol 210: 279-289, 2007.

6. Pichler M and Calin GA: MicroRNAs in cancer: From developmental genes in worms to their clinical application in patients. $\mathrm{Br}$ J Cancer 113: 569-573, 2015.

7. Sassen S, Miska EA and Caldas C: MicroRNA: Implications for cancer. Virchows Arch 452: 1-10, 2008.
8. Iorio MV, Visone R, Di Leva G, Donati V, Petrocca F, Casalini P, Taccioli C, Volinia S, Liu CG, Alder H, et al: MicroRNA signatures in human ovarian cancer. Cancer Res 67: 8699-8707, 2007.

9. Chen J, Wang M, Guo M, Xie Y and Cong YS: miR-127 regulates cell proliferation and senescence by targeting BCL6. PLoS One 8: e80266, 2013.

10. Guo LH, Li H, Wang F, Yu J and He JS: The tumor suppressor roles of miR-433 and miR-127 in gastric cancer. Int J Mol Sci 14: 14171-14184, 2013.

11. Resnick KE, Alder H, Hagan JP, Richardson DL, Croce CM and Cohn DE: The detection of differentially expressed microRNAs from the serum of ovarian cancer patients using a novel real-time PCR platform. Gynecol Oncol 112: 55-59, 2009.

12. Zhang L, Volinia S, Bonome T, Calin GA, Greshock J, Yang N, Liu CG, Giannakakis A, Alexiou P, Hasegawa K, et al: Genomic and epigenetic alterations deregulate microRNA expression in human epithelial ovarian cancer. Proc Natl Acad Sci USA 105: 7004-7009, 2008.

13. Calin GA and Croce CM: MicroRNA signatures in human cancers. Nat Rev Cancer 6: 857-866, 2006.

14. Saito Y, Liang G, Egger G, Friedman JM, Chuang JC, Coetzee GA and Jones PA: Specific activation of microRNA-127 with downregulation of the proto-oncogene BCL6 by chromatin-modifying drugs in human cancer cells. Cancer Cell 9: 435-443, 2006.

15. Horn H, Ziepert M, Becher C, Barth TF, Bernd HW, Feller AC, Klapper W, Hummel M, Stein H, Hansmann ML, et al; German High-Grade Non-Hodgkin Lymphoma Study Group: MYC status in concert with BCL2 and BCL6 expression predicts outcome in diffuse large B-cell lymphoma. Blood 121: 2253-2263, 2013.

16. Kramer MH, Hermans J, Wijburg E, Philippo K, Geelen E, van Krieken JH, de Jong D, Maartense E, Schuuring E and Kluin PM: Clinical relevance of BCL2, BCL6, and MYC rearrangements in diffuse large B-cell lymphoma. Blood 92: 3152-3162, 1998. 